\title{
An Active Recognition of Handwritten Isolated Arabic Characters
}

\author{
Youssef IGUIDER* and Makoto YASUHARA*
}

This paper introduces a new approach for recognizing cursively handwritten isolated Arabic characters. The approach is based on an active analysis of human handwriting movements, which was proposed and discussed extensively by the authors in a previous paper ${ }^{1)}$. The handwriting process is modeled through autoregressive moving average(ARMA) model. Model Reference Adaptive Control System (MRACS) theory is applied, in order to extract control pulses of the handwriting movements. The control pulses, which describe in details the handwriting movement's behavior, are then used for recognizing the shape of the cursively handwritten character. Although the presented approach is proposed for recognizing handwritten Arabic characters, it can, however, be applied equally well to actively recognize any other cursively handwritten scripts.

Key words : Arabic characters recognition, cursive handwritten character recognition, active analysis/recognition, modeling

\section{Introduction}

Recognition system for typewritten text are practically available for several latin based languages, mainly for English. However, systems for handwritten text still impose several constraints on writers to achieve acceptable recognition rates. Besides, cursive script recognition remains one of the most challenging unsolved problems in the field of character recognition.

On the other hand, in spite of the progress of recognition techniques for Latin, Kana and Chinese characters during the past three decades, the recognition of Arabic characters has remained almost untouched. Although Arabic characters are used in writing many widespread languages (Arabic, Persian(Farsi), Urdu, etc....), only few works have been published so far about handwritten Arabic characters recognition ${ }^{4) ~ 9)}$. The recognition systems proposed in these works were generally based on matching the characteristics related to the geometrical properties of the strokes.

The present paper introduces a new approach for recognizing cursive handwritten isolated Arabic characters. This approach is based on an active analysis

\footnotetext{
* University of Electrocommunications, Chofu

(Received March 6, 1995)

(Revised February 16, 1996)
}

of handwriting movements, which was proposed and discussed in depth by the authors in a previous paper ${ }^{1)}$. The handwriting process is modeled through an autoregressive moving average (ARMA) model. Model Reference Adaptive Control System(MRACS) theory is applied to extract control pulses of the handwriting movements. The control pulses, which describe in detail the handwriting movement, are then used for recognizing the shape of cursive handwritten characters. Although the presented approach is proposed for recognizing handwritten Arabic characters, it can be applied equally well to recognize any other cursive handwritten scripts.

\section{Active Analysis of the Handwriting Movement}

The content of this section is a brief review of the active recognition scheme proposed and discussed extensively by the authors in the reference ${ }^{1)}$.

\section{1 Fundamental Concept}

One of the traditional and common cases for understanding handwritten characters is gathering and analyzing statistics related to their variations in shapes, slants, stroke numbers, orders, directions and so forth, which we have called as passive analysis. Another insight for understanding them may come from modeling the human handwriting generation 
and studying the perceptual aspects of the generated handwriting samples, which we call here as active analysis in contrast to the previous one. Figure 1 shows the human handwriting process, which includes 4 basic levels of signal representations: 1 symbol, 2 . motor command, 3. pencil-point movement and 4. handwritten character. For generating handwritten characters, we make a forward trace through the neurological and dynamical transformations from the top to the bottom level to have the characters in the final form. The recognition of cursive handwritten characters can be seen as an inverted process of generation. We say that the recognition system is active if and only if it does involve in itself some generation process of the patterns to be recognized, otherwise passive.

\section{2 Handwriting System}

Figure 2 shows our basic idea: the systemtheoretic representation of the handwriting process. The system has no feedback involved at all and is supposed to be driven by a hypothetical input signal - control pulse, to give the handwriting signal at its output. To model the handwriting process, handwriting system is considered to be divided into two subsystems, muscular system $M$ and hand system $H$.

The transfer function of the muscular system could be modeled by a moving average(MA) process, the all-zero model : $B\left(k, z^{-1}\right)$. This is because the effects of both the inter- and intra-muscular frictions are expect-

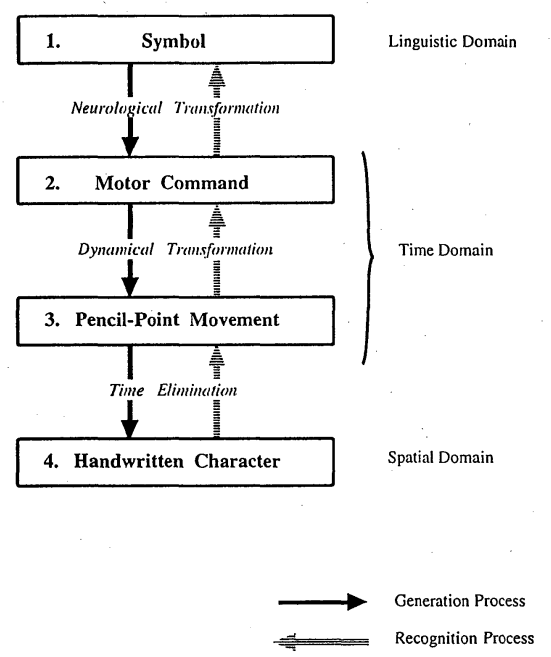

Fig. 1 Human handwriting process ed to result in certain energy absorption in some particular range of frequency components of the signal output from the muscular system. And this makes the muscular system $\mathrm{M}$ to be expressed by a kind of filter that receives the control pulse at the input and gives the muscular force at the output (Fig. 2).

The hand system could be expressed by an autoregressive (AR) model, having the transfer function with all-pole: $1 / A\left(k, z^{-1}\right)$. Thus the input-output relation of the overall handwriting system can be expressed by an Auto-Regressive Moving Average (ARMA) model having the following form:

$$
\begin{aligned}
y(k+1)= & \frac{B\left(k, z^{-1}\right)}{A\left(k, z^{-1}\right)} v(k)=H\left(k, z^{-1}\right) v(k), \\
A\left(k, z^{-1}\right)= & 1+a_{1}(k) z^{-1}+a_{2}(k) z^{-2}+\cdots+a_{m}(k) z^{-m}, \\
B\left(k, z^{-1}\right)= & b_{0}(k)+b_{1}(k) z^{-1}+b_{2}(k) z^{-2}+\cdots \\
& +b_{n}(k) z^{-n}
\end{aligned}
$$

$v(k)$ and $y(k+1)$ is the input control pulse and the output handwriting signal, respectively. $z^{-1}$ denotes the operator of a unit time delay.

After estimating the parameters of both $A R$ and MA of the ARMA model, it becomes possible to extract the control pulses of the handwriting movements by applying the MRACS theory. The way to extract the control pulses is discussed in detail in the reference $^{1)}$

\section{3 Control Pulse}

The movements involved in the actual handwriting are so much complicated that an attempt to interpret them in terms of a simplified model might be point less if we are to make precise physiological investigations about the actual human handwriting process. However, it must be rather contributory to our present studies to take a simplified model into account. To begin with, we consider the following two assumptions $^{3)}$ : (1) the handwriting movements are resolved into three principal components, namely, $X$,

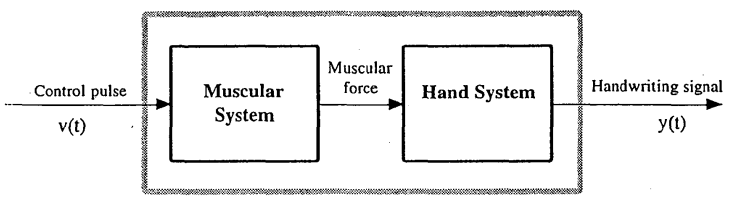

Fig. 2 System-theoretic representation of handwriting process 
$Y$ and $Z$ directions (the $Z$-component, writing pressure, is neglected for simplification), (2) the muscles involved in each component of the movements are grouped together into a pair to cause them to generate the forces to antagonistic directions having the prescribed magnitude and timing's. Under these basic assumptions, we introduce the definition of control pulse, which plays an essential role in this study.

The cursive characters are written by a rapid sequence of motions caused by different applications of the magnitude and timing's of muscle activities. It is shown experimentally in the reference ${ }^{3)}$ that the shapes of characters are coded only in time, i.e., by the durations, not by the magnitude of muscle contraction. It is also shown that the magnitude of the force determines the general size of the characters written. We, therefore, assume that the control signal of the handwriting can be represented by the square waveform having three magnitude levels, which we call control pulses (Fig. 3). The level 1 and level -1 mean ON activities of the group of muscles to the positive and the negative directions, respectively, and 0 means OFF activity (abbreviated in later as $O N$-pos, ON-neg and OFF). $T_{i}^{\prime}$ 's are controltiming's at which the muscular activity changes from one level to another. It is the control timing information (not the magnitude) of the force used that we do need to extract from the original handwritten characters.

We think of the control pulses to be the representation of the handwriting movement at the motor command level shown in the Fig. 1. They are expected to be more direct representations, namely, at the higher level of handwriting process, than are the pencil-point

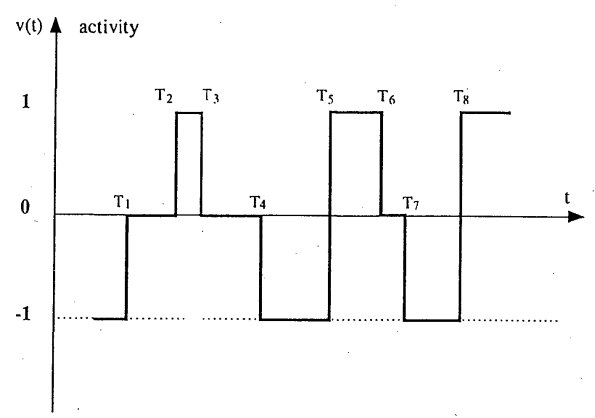

Fig. 3 Control pulses sequence of handwriting movement movements. The control pulses are then expected to describe in detail the behavior of the handwriting movements. Thus the extracted control pulses makes it easy for us to catch which characters are being written.

\section{Properties of the Arabic Characters}

Arabic script is always written cursively from the right to the left. Arabic alphabet (Fig. 4) has many differences from other systems of characters. The main characteristics of Arabic characters are as follows:

- Dot is one of the main features of the Arabic characters. Most characters involve a main stroke (body of the character) and complementary one or more dots (up to three dots). These dots can be located below or above the body of the character.

- Other than the dots mentioned above, some characters contain a secondary stroke, which may be a vertical line or a zigzag mark.

- Width and length of the Arabic characters differ from one character to another.

- Some isolated Arabic characters are written in a single stroke with no need to lift up the pen.

- Many characters have exactly the same body (longest continuous portion of the character which is written before lifting up the pen) ; however, they are distinguished from each other by the additional dots or other secondary strokes.

\section{Approach to Active Recognition}

This section assumes that control pulses related to considered handwritten characters have already been extracted for both $X$ and $Y$ axis. Further, to simplify the notations, we will use $x$-pulses and $y$-pulses, to indicate "control pulses corresponding to the $X$ axis" and "control pulses corresponding to the $Y$ axis", respectively.

The approach starts by classifying a considered character according to the number of strokes involved in it. By stroke we mean portion of the character that is written without lifting up the penpoint from the surface of the writing tablet. This way, the whole set of isolated Arabic characters can be divided into four subsets: namely the characters consisting of one, two, three and four strokes. 


\begin{tabular}{|c|c|c|c|}
\hline No. & Character & Shape & Sequence \\
\hline 1 & Alif & 1 & $\mu^{0}$ \\
\hline 2 & $B a$ & 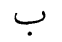 & $\underbrace{\prime \prime}$ \\
\hline 3 & $T a$ & ت & $\underbrace{\oplus \cdots \infty}$ \\
\hline 4 & Tha & $\ddot{ت}$ & $\underbrace{p \cdot P_{0}}_{0}$ \\
\hline 5 & Jim & $\tau$ & ${ }_{0}^{\infty} \vec{C}_{0}$ \\
\hline 6 & $\mathrm{Ha}$ & $\tau$ & $\Phi_{\vec{r}}$ \\
\hline 7 & Kha & $\dot{\tau}$ & $\vec{r}^{-\infty}$ \\
\hline 8 & Dal & J & נi \\
\hline 9 & Dhal & j & $j_{j}^{\infty}$ \\
\hline 10 & $R a$ & $J$ & נ, \\
\hline 11 & Zayn & $j$ & $\ddot{j}^{-\infty}$ \\
\hline 12 & Sin & س & $\underset{\Phi=\infty}{\infty}$ \\
\hline 13 & Shin & ش & 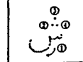 \\
\hline 14 & $\dot{s} a d$ & $ص$ & $\bigcup_{0}^{\infty}$ \\
\hline 15 & Dad & $\dot{~}$ & $\underbrace{\oplus \vec{\Phi}}_{\Phi=0}$ \\
\hline
\end{tabular}

\begin{tabular}{|c|c|c|c|}
\hline No. & Character & Shape & Sequence \\
\hline 16 & $\dot{T} t a$ & $b$ & gुg \\
\hline 17 & D.ha & b & $\stackrel{\phi b}{-\infty}$ \\
\hline 18 & Ayn & $\varepsilon$ & ic \\
\hline 19 & Ghayn & $\varepsilon$ & 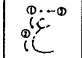 \\
\hline 20 & $F a$ & G & $\underbrace{-\oplus}$ \\
\hline 21 & $\dot{Q} a f$ & $\ddot{~}$ & 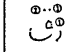 \\
\hline 22 & Kaf & s] & $\underbrace{j}_{j}$ \\
\hline 23 & Lam & $J$ & נן \\
\hline 24 & Mim & $\rho$ & $\int_{0}^{8}$ \\
\hline 25 & Nun & $\dot{U}$ & ${ }^{\infty}$ \\
\hline 26 & Hha & 0 & $\Delta_{\omega}^{2}$ \\
\hline 27 & Waw & 9 & $\Phi \hat{9}$ \\
\hline 28 & $Y a$ & ي & $\underbrace{\infty}_{\infty \rightarrow \infty}$ \\
\hline 29 & - Hamza & $\varepsilon$ & $\Phi^{\mathcal{E}}$ \\
\hline 30 & Ta-Marbuta & $\ddot{0}$ & $\begin{array}{r}0 . . \Phi \\
\text { oi }\end{array}$ \\
\hline
\end{tabular}

Fig. 4 Isolated Arabic characters

Further, the system classifies the handwritten characters inside each of the four subsets by using several features in a sequential form. Let us now consider these features

\section{1 Time-duration of Control Pulses}

To investigate promptly the shape of a considered handwritten character, the system focuses on certain parts of $x$-pulses and/or $y$-pulses (see Fig. 5). Behavior of the handwriting movement represented by these control pulses is compared with fragments that distinguishes the shape of a character-candidate. The behavior of a handwriting movement is analyzed by estimating the time-duration (TD) related to each of the 3 muscular activities, namely $O N-p o s, O N-n e g$ and $\mathrm{OFF}$, inside of the focused group of control pulses:

$$
\begin{aligned}
& T D_{\text {ON-pos }}=\sum_{i=f}^{l} O N-\text { pos }, \\
& T D_{\text {ON-neg }}=\sum_{i=f}^{l} O N-n e g, \\
& T D_{\text {OFF }}=\sum_{i=f}^{l} O F F,
\end{aligned}
$$

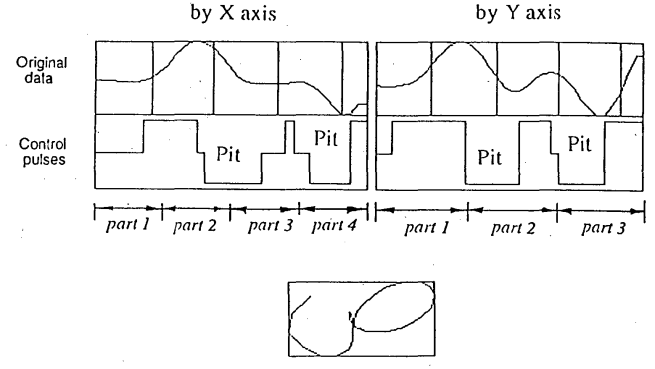

Fig. 5 Investigating control pulses of sample Sad

$f$ and $l$ are, respectively, the first and the last pulses of the focused group of control pulses. By comparing $T D_{O N-p o s}, T D_{O N-n e g}$ and $T D_{O F F}$, within the focused part of $x$-pulses and/or $y$-pulses, the system determine the orientation of the handwriting movement.

Figure 5 shows $x$-pulses and $y$-pulses representing the handwriting movement that is performed to shape the character $\bar{S} a d$. Note that the first(left) part of $y$-pulses is clearly dominated by ON-pos. Domination of ON-pos should meet the following: 


$$
\left\{\begin{array}{l}
T D_{O N-\text { pos }}>T D_{\text {ON-neg }}, \\
T D_{O N-\text { pos }}>T D_{\text {OFF }}
\end{array}\right.
$$

This shows that the handwriting movement, represented by the first part of $y$-pulses, was oriented to the positive direction of $Y$ axis, towards drawing the first fragment of the character $\bar{S} a d$.

Such investigations allow a detailed understanding of the nature of the handwriting movements that led to the shaping of different fragments of a considered character.

\section{2 Pit}

We call Pit-a group of $\mathrm{ON}-$ neg control pulses which exists between two groups of ON-pos and/or OFF control pulses. Pit represents the changing of a handwriting movement's direction from the positive to the negative direction, and back. This double change of the handwriting movement results in having a minima on the drawn shape.

Arabic script is mostly written cursively through semi-circular movements oriented "down $\rightarrow$ left $\rightarrow$ $u p$ ". Thus the number of these Pits, especially by $Y$ axis, helps recognizing the shape of the considered characters. Figure 5 shows 2 Pits at $x$-pulses and 2 Pits at $y$-pulses.

\section{3 Ratio of ARMA Parameters}

The parameter $b_{0}$ (Eq. 3), which was estimated while modeling the handwriting system, should be available at this step. Ratio between the parameters $b_{0}$ related to $X$ and $Y$ signals gives an idea about the proportion between the width and the length of the corresponding handwritten character.

\section{Recognition Algorithm}

This section presents an algorithm for recognizing handwritten isolated Arabic characters. This algorithm makes use of all the features discussed above.

As mentioned before, first, the system starts by looking up the number of strokes involved in the character which is currently considered for recognition. According to this, a current character may be classified as a one-, two-, three- or four-strokes character (Fig. 6).

Further, the system classifies the considered handwritten characters inside each of the four subsets (one-, two-, tree- and four-strokes subsets).

\subsection{One-stroke Characters}

The set of one-stroke characters includes twelve characters (Alif, Ha, Dal, Ra, Sin, Sad, Ayn, Lam, Mim, Hha, Waw and Hamza). These characters are divided into two groups, depending on the number of Pits in $y$-pulses (Fig. 7) :

1. The first group includes characters $\operatorname{Sin}, \bar{S} a d$, Ayn, Ha, Waw, Hamza and Mim. The $y$-pulses for these characters should involve more than one Pit. To write a character from this group, the handwriting movement should change its direction more than once by $Y$ axis.

2. The second group includes characters Alif,

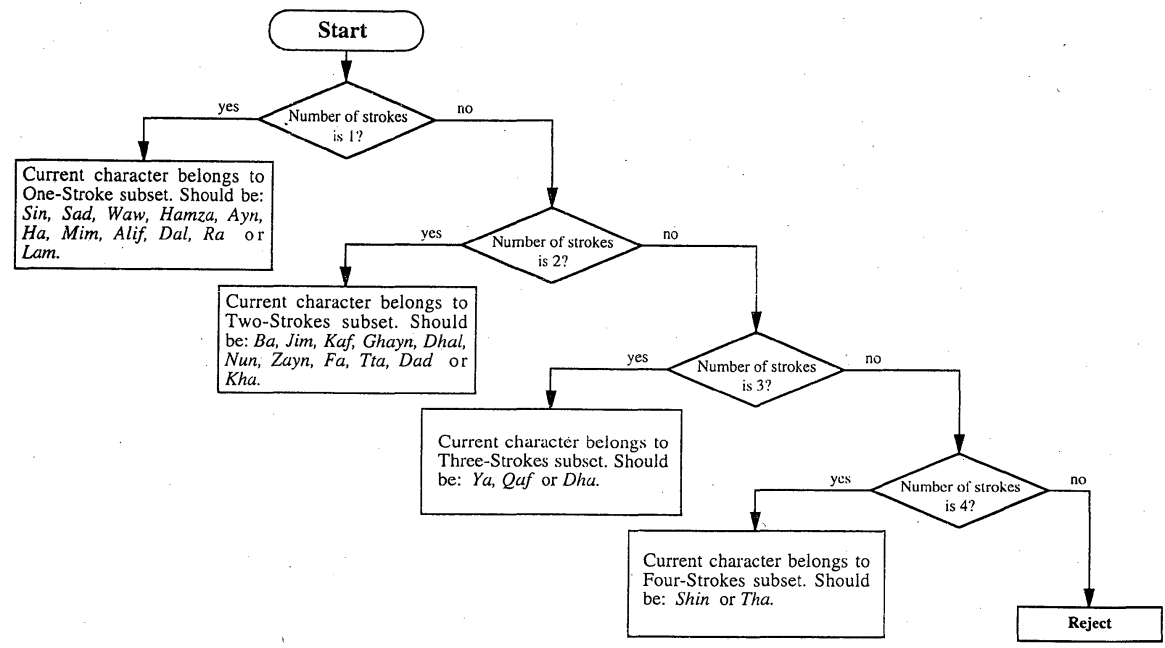

Fig. 6 Classifying characters by the number of involved strokes 


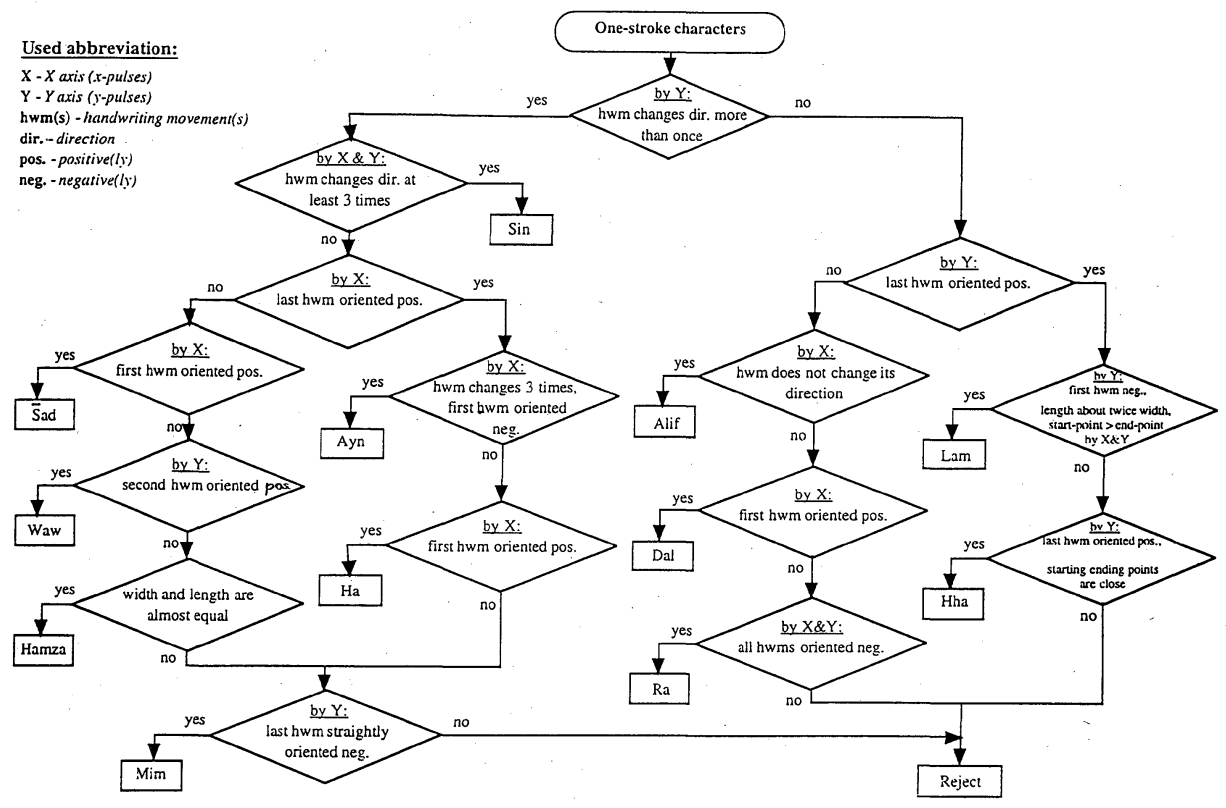

Fig. 7 Classifying one-stroke characters

Dal, Ra, Lam and Hha, y-pulses of which should involve at most one Pit. To write a character from this group, the handwriting movement should change its direction by $Y$ axis at most once.

Further, one-stroke characters are classified within each of these two groups.

\section{1. 1 First group of one-stroke characters}

As shown in Fig. 7 , if the $y$-pulses involve 3 Pits, i.e., the handwriting movement changes its orientation down-up 3 times, then this character should be Sin. Indeed, the shape of the character $\operatorname{Sin}$ includes 3 distinguishing minima along $y$-axis.

If the above condition is not satisfied, the system focuses the last part of $x$-pulses. If, in this part, $\mathrm{ON}$ pos is dominating (i.e., the condition in the Eq. 7 is satisfied), then the considered character could be Ayn or Ha. This is because the last part of the shape is written with a handwriting movement which is positively oriented by $X$ axis. The characters $\bar{S} a d$, Waw and Hamza meet the opposite situation. Meanwhile, the character Mim may be found in both situations. Because, to draw the last part of this character, the handwriting movement should be oriented straightly downward(negatively by $Y$ axis). However, this handwriting movement might shift slightly to the right or the left, leading to the generation of extra ON-pos or ON-neg.

In the above first case, $A y n$ and $H a$ may be differentiated based on how the first movement starts. To write a Ayn, the first movement should be oriented to the negative direction by $X$ axis. Whereas it should be oriented to the positive direction for the character $\mathrm{Ha}$. Moreover, $x$-pulses corresponding to a Ayn should involve 2 Pits.

As for the second case, to differentiate the character $\bar{S} a d$ from Waw and Hamza, the system investigates the handwriting movement used to draw the first half part of the character body. This handwriting movement should be oriented, for a long while, positively to $X$ axis. Therefore, $O N$-pos should be dominating in the first half part of $x$-pulses.

If the above condition is not satisfied, moreover the middle part of $y$-pulses involves a relatively long time-duration of $O N$ - $p o s\left(T D_{O N-p o s}\right)$, then the system should be dealing with the character Waw. To draw the central part of a Waw, the handwriting movement should be oriented to the positive direction by $Y$ axis.

If all the conditions imposed so far are not satisfied, meanwhile the width and the length of the character are stretching to be equal, then the considered handwritten character could probably be a Hamza. 
The character Mim has some similarity with Waw. However it can be differentiated based on how the second half part of the shape should be drawn. IN this case, $y$-pulses corresponding to the second half part of the Mim should be totally dominated by $O N$ neg.

\subsubsection{Second group of one-stroke characters}

This group includes characters $y$-pulses of which involve at most one Pit. To write a character from this group, the handwriting movement should change its direction at most once by $Y$ axis.

This group is also divided into two subgroups depending on the last performed handwriting movement. The first subgroup includes characters written with a final handwriting movement which is oriented positively by $Y$ axis. The last part of $y$-pulses should be dominated by $O N$-pos. This subgroup includes the characters Lam and Hha.

The opposite situation corresponds to the second subgroup. IN this case, the last part of $y$-pulses should be dominated by $O N$-neg. This subgroup includes characters Alif, Dal and $R a$.

Concerning the first subgroup, if the spatial length of the character is almost twice as its width; and the drawing starts at a higher level than where it finishes (by both $X$ and $Y$ axes) ; moreover the handwriting movement, which shapes the first half part of the character, is oriented straightly downward, then the current character should be a Lam.

As for the character Hha, the drawing should ends near where it starts (by both $X$ and $Y$ axes). Moreover, the corresponding handwriting movement should be oriented positively by $X$ axis at the beginning as well as at the end of the drawing.

Now let us consider the second subgroup. Here, the system begins by checking up Alif's symptom. In this case, the handwriting movement should not considerably change its direction by $X$ axis, during the whole time of drawing.

If Alif's symptoms is not matched, then the next character to be considered is Dal. Here, the handwriting movement behind thefirst fragment of the character should be oriented mainly to the positive direction by $X$ axis. Thus, the first half part of $x$-pulses should be dominated by $O N$-pos.

The investigated character could be a $R a$, if its

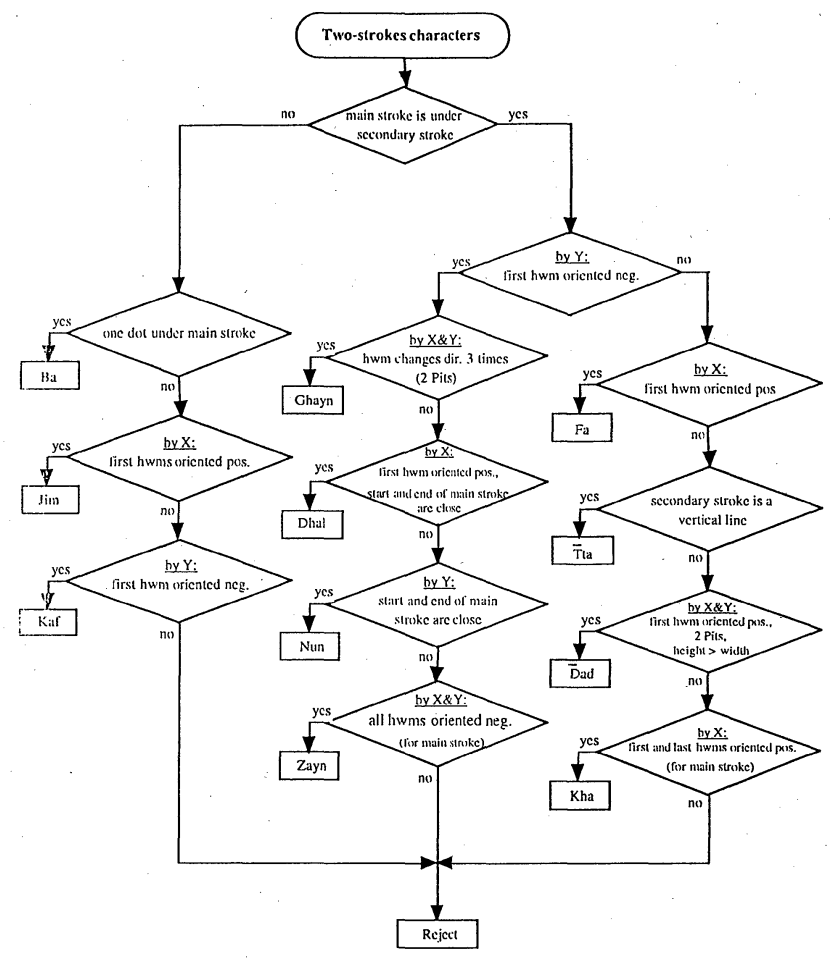

Fig. 8 Classifying two-strokes characters 


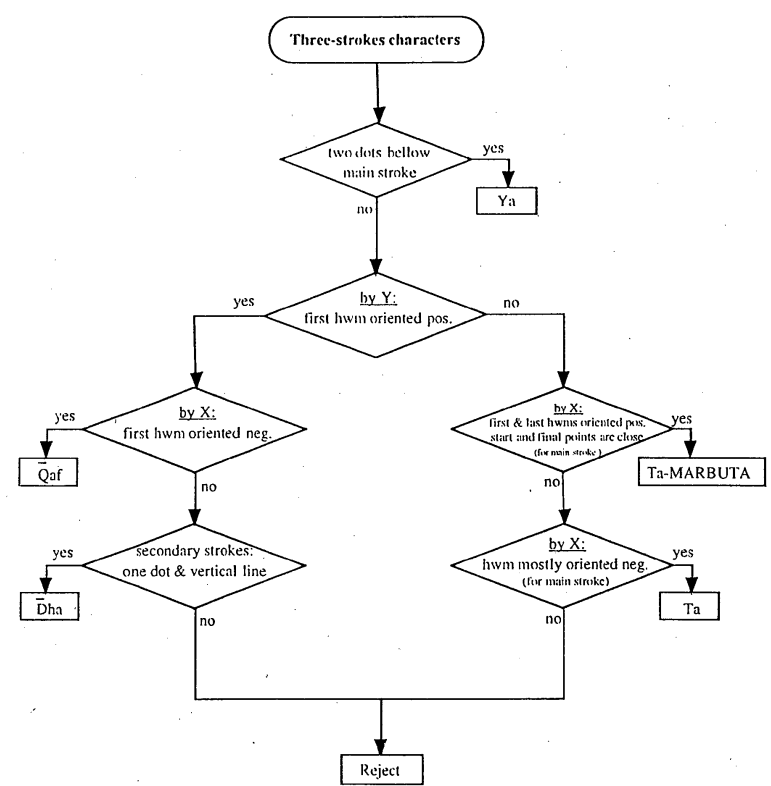

Fig. 9 Classifying three-strokes characters

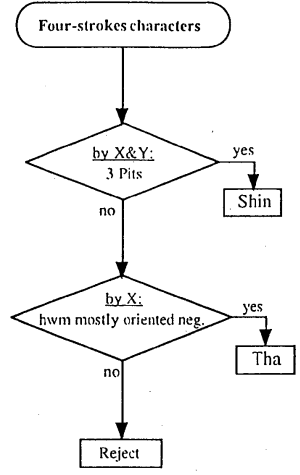

Fig. 10 Classifying four-strokes characters corresponding handwriting movement is oriented all the time negatively by both $X$ and $Y$ axes. In this case, both $x$-pulses and $y$-pulses should be dominated by $O N-n e g$.

At the end, the currently considered one-stroke character is rejected, if it does not match any of the conditions considered so far.

\subsection{Other subsets}

Further, as shown in the Fig. 8-10, the system uses the same approach to deal with the other subsets (two-, three- and four-strokes isolated Arabic characters).

\section{Results and Comments}

The algorithm presented above has been developed after observing and investigating a large number of training samples of handwritten Arabic characters. Both $x$-pulses and $y$-pulses as well as other features have been studied extensively.

\section{Experimental results and comments}

Computer experiments were performed for all Arabic characters shown in Fig. 4.2400 different samples were used to carry out these experiments; 80 handwritten samples for each Arabic character. Results of the experiments showed an average recog- nition rate of $98.6 \%$ (Fig. 11).

Inaccuracy in writing was the main cause behind the misrecognized and rejected samples. However, very slow handwriting was also another cause. The recognition system presented here is actually based on our previously published work ${ }^{11}$, which focuses on fast handwriting. By fast cursive handwriting we mean highly practiced and skilled movements of handwriting which are executed by means of a rapid sequence of motions without instantaneous position feedback ${ }^{1}$.

Let us consider some similar works. Reference 4) proposed a structural recognition method making use of geometrical and topological properties of the script, and performed a recognition rate of $91 \%$. Reference 5) classified characters using syntactic strings of primitive directions in the plane, and reported a recognition rate of $93 \%$. Meanwhile ${ }^{6}$ reported a recognition rate of $95.4 \%$, using a structural classification based on coordinates of the strokes. Reference 7) based its classification on a syntactic representation of the characters using geometrical primitives like curves and strokes, but no recognition rate was reported. The highest rate (100\%) was reported by Refs. 8) and 9), where the classifications were based on direction codes and other geometrical 


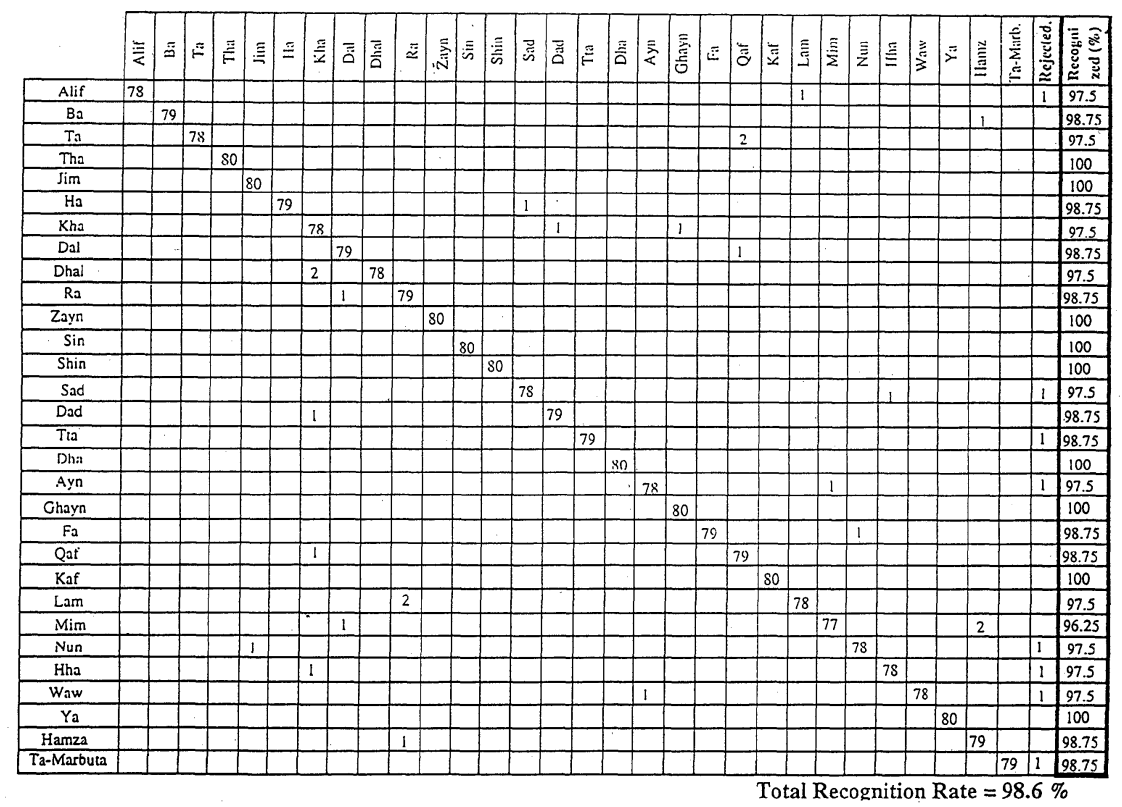

Fig. 11 Recognition results

features.

Note that the significance of the present work is, however, not only the satisfactory recognition rate which is $98.6 \%$, but the fact is it also attempts to introduce a totally new concept for dealing with handwritten scripts. As it bases its approach on active analysis of handwriting movements, by application of a new way of modeling the handwriting process.

\section{Threshold element}

The best SDR value in the reference ${ }^{1)}$ was searched around the threshold value $\theta=0.6120$, the 3-level Gaussian optimum quantizer. However, in this work we used the threshold $\theta=1$, in order to have the decreased number of small extra pulses (see the following comment).

\section{Comments on the sensitivity}

To demonstrate the high sensitivity of the system, Fig. 12 considers a cases where it is rather difficult to recognize the handwritten sample ( $\operatorname{Sin}$ ) just by its original $x$ and $y$ data. The 3 vertical maxima characterizing this Arabic character can hardly be noticed by the original data of $Y$ axis(Fig. 12). Meanwhile, these same 3 maxima can be easily noticed through

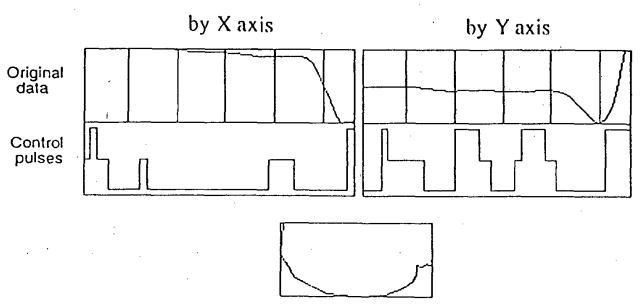

Fig. 12 High sensitivity of the system

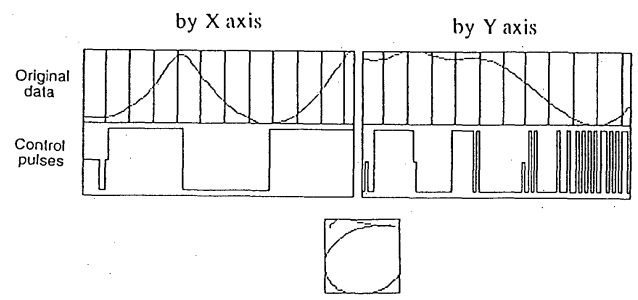

Fig. 13 More on the sensitivity

the control pulses ( $Y$ axis).

To have a better idea about the sensitivity of the system. Figure 13 demonstrates system's ability to perceive (when it is necessary) even very small deviations of handwriting movements. The hand vibrations result in generating extra small pulses. Note that the small vibrations are hardly perceived through the original data. Actually this demonstra- 
tion was performed intentionally on a sample written slowly ( 2.8 seconds) using a threshold $\theta=0.62$ The extra small pulses are considered to be caused by a visual feedback involved while the sample has been written slowly.

\section{Conclusion}

In this work, we have introduced a new solution toward recognizing handwritten isolated Arabic characters. The proposed approach is based on the active analysis of human handwriting movements. The handwriting process is modeled through an autoregressive moving average(ARMA) model. Model Reference Adaptive Control System (MRACS) theory is applied to extract control pulses of the handwriting movements. The control pulses are then used to recognize the shape of cursive handwritten characters.

Finally, it must be kept in mind that it is not necessary for the recognition of handprinted characters to apply such a complicated active method and to extract signals at the higher level of the handwriting process. For handprinted characters a simpler and straightforward method based on passive analysis can be used successfully.

Although the presented approach is proposed to recognize handwritten Arabic characters, it can, however, be applied equally well to recognize any other cursive handwritten scripts.

\section{References}

1) Y. Iguider and M. Yasuhara: Extracting Control Pulses of Handwriting Movement-Toward an Active Recognition of Cursive Handwritten Characters-, Trans. of the Society of Instrument and Control Engineers, 31-8, 1175/1184 (1995)

2) Y. Iguider and M. Yasuhara: A Control-Theoretic Description of Handwriting Process, Preprints of The 24th ISCIE Symposium On Stochastic Systems Theory and Its Applications, 45/48 (Nov. 1992)

3) M. Yasuhara: Experimental Studies of Handwriting Process, Rep. Univ. Electro-Comm. 25-2 (Sci. \& Tech. Sect.), 233/254 (Mar. 1975)

4) H. Almuallim and S. Yamaguchi : A Method of Recognition of Arabic Cursive Handwriting, IEEE Trans. on Pat. Anal. and Mach. Int., PAMI-9-5, 715/722 (Sep. 1987)
5) M.S. El-Wakil and A.A. Shoukry: On-Line Recognition of Handwritten Isolated Arabic Characters", Pattern Recognition, 22-2, 97/105(1989)

6) A. Amin, A. Kaced, J-P. Haton and R. Mohr. "Hand Written Arabic Character Recognition by the I. R. A. $C$. System, 5th International Conference on Pattern Recognition, Miami, Florida, 729/731 (Dec. 1980)

7) A.Amin, G. Massani and J-P. Haton: Recognition of Handwritten Arabic Words and Sentences, IEEE, CH 2046-1/84, 1055/1057 (1984)

8) S. Al-Emami and M. Usher: On-Line Recognition of Handwritten Arabic Characters, IEEE Trans. on Pat. Anal. and Mach. Int., 12-7 (Jul, 1990)

9) T.S. El-Sheikh and S.C. El-Taweel : Real-Time Arabic Handwritten Character Recognition, Pattern Recognition, 23-12, 1323/1332 (1990)

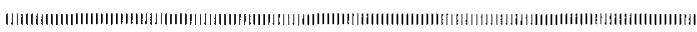
Youssef IGUIDER

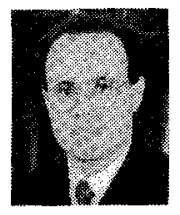

Was born in Morocco in 1961 . He received the M. Sc. in Electrical Engineering from the Odessa Polytechnic Institute in USSR in 1987. He is involved in the Doctoral program with the Department of Computer Science and Information Mathematics at the University of Electro-Communications in Tokyo since April 1990. Currenty he is on leave to work with the R\&D Center at Kyushu Matsushita Electric co. His research interests include handwriting recognition and its applications.

\section{Makoto Yasuhara (Member)}

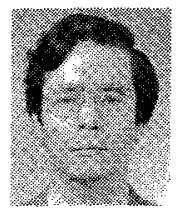

Was born in Tokyo in 1939. He received the B.S. from the University of ElectroCommunications in 1963, and the Doctors of Medical Science and Engineering in 1974 and in 1986, respectively, both from the University of Tokyo. He joined the University of ElectroCommunications in 1966 and was a professor of the Department of Computer Science and Information Mathematics from 1987 to 1993 and currently a professor of the Department of Information Manegement Science, the Graduate School of Information Systems. His research interests include the stochastic system theory, the control theory and their applications. $\mathrm{He}$ is a member of the IEEE, the IEICE, the IPSJ and the SICE. 